\title{
Mutational signatures in colon cancer
}

\author{
Priyatama Pandey ${ }^{1}$, Zhi Yang ${ }^{1}$, Darryl Shibata ${ }^{2}$, Paul Marjoram ${ }^{1}$ and Kimberly D. Siegmund ${ }^{1 *}$ (D)
}

\begin{abstract}
Objective: Recently, many tumor sequencing studies have inferred and reported on mutational signatures, short nucleotide patterns at which particular somatic base substitutions appear more often. A number of signatures reflect biological processes in the patient and factors associated with cancer risk. Our goal is to infer mutational signatures appearing in colon cancer, a cancer for which environmental risk factors vary by cancer subtype, and compare the signatures to those in adult stem cells from normal colon. We also compare the mutational signatures to others in the literature.

Results: We apply a probabilistic mutation signature model to somatic mutations previously reported for six adult normal colon stem cells and 431 colon adenocarcinomas. We infer six mutational signatures in colon cancer, four being specific to tumors with hypermutation. Just two signatures explained the majority of mutations in the small number of normal aging colon samples. All six signatures are independently identified in a series of 295 Chinese colorectal cancers.
\end{abstract}

Keywords: Somatic mutations, Mutational process, Topic model, Latent Dirichlet allocation model

\section{Introduction}

The first large study of somatic mutations in cancer identified 20 mutational signatures in 7042 primary tumors from 30 different classes [1]. They defined mutational signatures by patterns of three consecutive nucleotides, including one base $3^{\prime}$ and one $5^{\prime}$ of the nucleotide substitution, and represented by a linear combination of the 96-possible three-base patterns. The mutational signatures were annotated and published in the Catalogue of Somatic Mutations in Cancer (COSMIC) database [2]. Four signatures were identified in 557 colorectal cancers [1], three signatures with probable associations attributed to one of the mechanisms of aging, DNA mismatch repair, or Pol $\epsilon$ mutation and the fourth of unknown origin.

A simple probabilistic model for mutational signatures, proposed shortly thereafter, assumed independent contributions (i.e., multiplicative probabilities) of the neighboring bases composing the nucleotide pattern [3].

\footnotetext{
*Correspondence: kims@usc.edu

${ }^{1}$ Department of Preventive Medicine, Keck School of Medicine of the University of Southern California, 2001 N. Soto Street, Los Angeles, CA 90032, USA

Full list of author information is available at the end of the article
}

This resulted in a more parsimonious model with fewer parameters and the ability to detect longer five-base signature patterns. A reanalysis of the same colon cancer data using this new probabilistic model also reported four mutational signatures, but their make-up was different. The previous Pol $\epsilon$ signature was split into two signatures, one favoring $\mathrm{C}>\mathrm{T}$ mutations at $\mathrm{TpCpG}$ and the second favoring $\mathrm{C}>\mathrm{A}$ at $\mathrm{TpTpCpT}$, a signature four bases in length. The remaining two signatures were attributed to aging, and unknown origin. Interestingly, the DNA mismatch repair signature was not reported.

Today, the number of single-base substitution signatures in the COSMIC database has increased to 49; seven of these signatures relate to DNA mismatch-repair (MMR) deficiency. Recent studies characterizing cancers with hypermutation [4] and cancers along the gastrointestinal tract $[5,6]$ reported multiple MMR signatures. A recent reanalysis of data from the Cancer Genome Atlas by Liu et al. identified six signatures in colon cancer [6], four of which are identified as occurring primarily in cancers with high mutational burden. We sought to understand the connection between these six mutational signatures and those found using the probability mutational signature model.

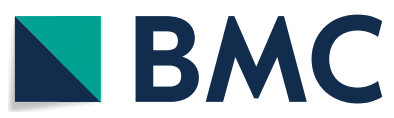

C The Author(s) 2019. This article is distributed under the terms of the Creative Commons Attribution 4.0 International License (http://creativecommons.org/licenses/by/4.0/), which permits unrestricted use, distribution, and reproduction in any medium, provided you give appropriate credit to the original author(s) and the source, provide a link to the Creative Commons license, and indicate if changes were made. The Creative Commons Public Domain Dedication waiver (http://creativecommons.org/ publicdomain/zero/1.0/) applies to the data made available in this article, unless otherwise stated. 
In addition to studying the variation in mutational signatures appearing in different subtypes of colon cancers, we investigated whether the mutational signatures differed across different time periods. We classified somatic mutations by their time of occurrence, occurring in the original tumor cell ('trunk' mutation) or appearing de novo during tumor growth ('branch' mutation), and compared their signatures to those found in adult stem cells from normal colon. We exploit publicly available data from a study of adult stem cells (ASCs) in normal colon [7], the Cancer Genome Atlas (TCGA), and the International Cancer Genomics Consortium (ICGC). Our analysis identifies six mutational signatures using ASCs and TCGA colon cancers that are validated in the ICGC Chinese colorectal cancers.

\section{Main text \\ Data}

Human adult stem cells (ASCs) from normal colon

Whole genome sequencing of 21 samples from 6 human ASCs from normal colon was performed and published in [7]. Processed somatic mutation data were downloaded from [8].

\section{TCGA colon adenocarcinoma (COAD-US)}

We downloaded somatic mutation data from 435 colon adenocarcinoma from the Genomic Data Commons Data Portal [9]. The tumor characteristic microsatellite instability (high, low, stable) was downloaded as part of the clinical data. A total of 431 samples with somatic mutation data had information on microsatellite instability. We obtained the variable on $\operatorname{Pol} \epsilon$ mutation from the supplementary data in [10]. We note that our downloading and filtering of the TCGA data resulted in notable differences from the previously analyzed data made available in $[1,3]$.

We classified mutations by their time of occurrence (trunk/branch) by applying the criteria of Williams et al. [11], using information on tumor purity and allele frequency. We restricted our data set to the COAD-US samples in [11] with purity $\geq 70 \%(\mathrm{n}=99)$, and classified the mutations with frequency $\geq 0.25$ as trunk and the rest as branch. After mutation classification, six samples with fewer than 10 mutations along with their tumor-matched sample were omitted from further analysis.

\section{Colorectal adenocarcinoma in China (COCA-CN)}

The somatic mutation data in Chinese colorectal adenocarcinoma were downloaded from the ICGC Data Portal [12]. This data set contains $2,941,990$ mutations in 295 Chinese colorectal samples.

See Additional file 1 for details on mutation filtering.

\section{Statistical methods}

We applied the probabilistic mutation signature model [3] to infer mutation signatures and their exposure frequencies in normal colon ASCs and COAD-US tumor samples. We restricted all samples to mutations on chromosomes 1-22 and fit the model using the pmsignature package in $R$ [3]. We specify the model for a five-base context and include the direction of the transcription strand (positive/negative). The four nucleotides flanking the substitution, two upstream and two downstream, are extracted from the reference genome. As the ASCs from normal colon and COAD-US samples were sequenced at different times and mapped to different reference genomes, flanking bases are extracted using the same reference to which the corresponding sample was mapped, (hg19 for ASC samples and hg38 for COAD-US). We selected the optimum number of latent mutational signatures by minimizing the Bayesian Information Criterion (BIC) and the bootstrap standard errors for the model parameters [3].

The Shiny app iMutSig [13] was used to compare our discovered signatures with the published mutational signatures from pmsignature and from the COSMIC mutational signature website $[2,3]$. iMutSig uses cosine similarity to compute the similarity of any two mutational signatures. When comparing our five-base signature to the three-base signature in COSMIC, we sum the probabilities of the signature vector from the fivebase model over the features unmeasured in the threebase model. Due to the independence assumption of our model, this is equivalent to a comparison using just the features shared in common by the two models.

Finally, we applied a hierarchical latent Dirichlet allocation model (HiLDA) [14] to test the equivalence of mutational signature exposures between trunk and branch mutations. We used the posterior distributions of the mean differences to test for differential exposures for any single signature (signature-level tests). The analysis was performed in $\mathrm{R}$ using the $H i L D A$ package.

\section{Results}

Mutational signature analysis was applied to 127,748 mutations from 431 COAD-US samples and 860 mutations from 6 normal colon ASCs. The highest numbers of somatic mutations are found in the MMR-deficient, MSI-H and Pol $\epsilon$ cancers (Additional file 1: Figure S1). We fit the probability mutation signature model for different numbers of mutational signatures (2 through 8 ) and using the criteria of low bootstrap error and low BIC, selected six mutational signatures as having the best fit (Additional file 1: Figure S2). 


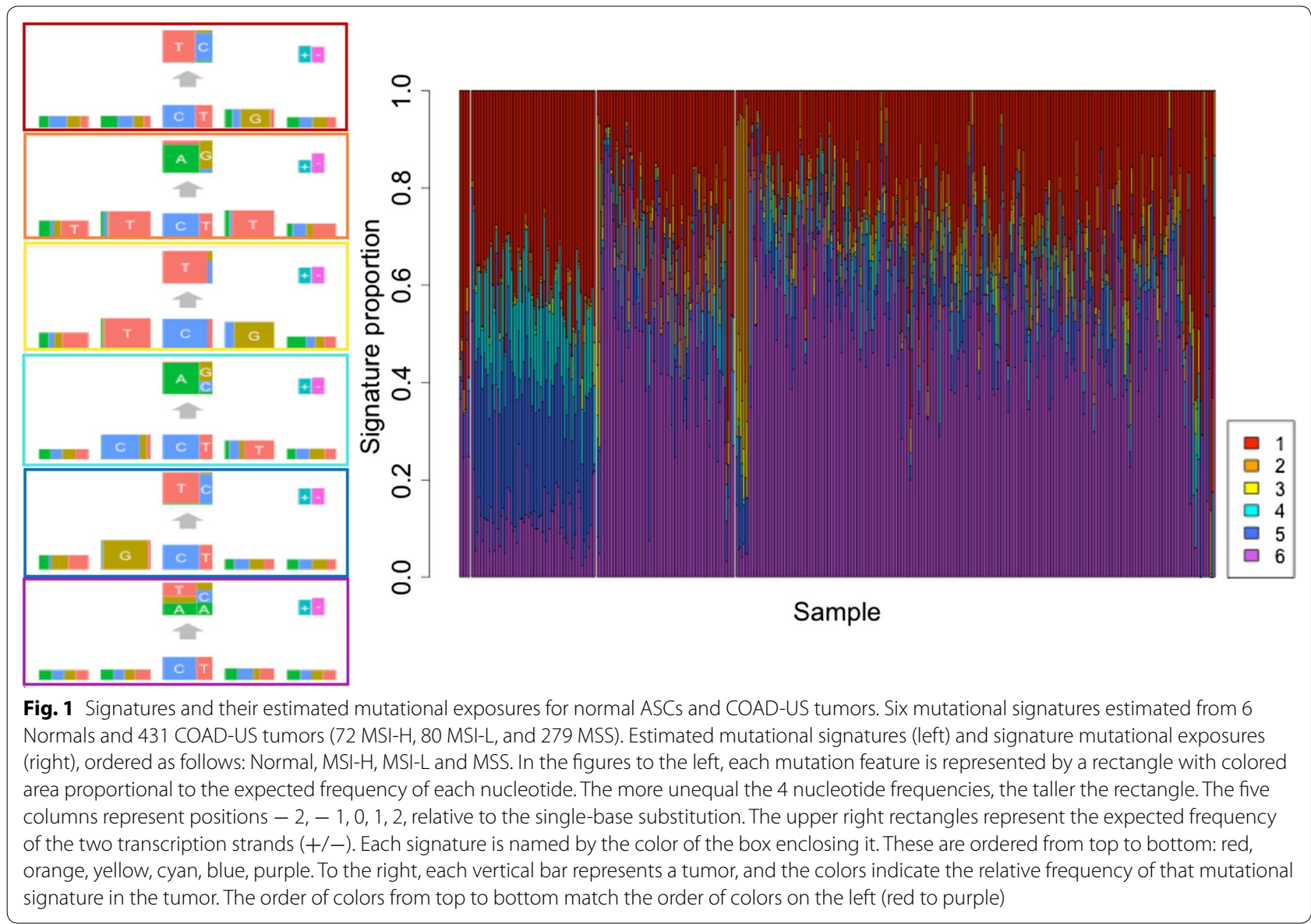

Figure 1 shows the six inferred mutational signatures along with the estimates of signature mutational exposures. The six signatures included the four signatures previously identified by Shiraishi et al. [3] (red, orange, yellow, purple). The red signature was described as being due to aging, whereas the orange and yellow signatures were described as being due to the deregulated activity of the polymerase Pol $\epsilon$, while purple was of unknown origin. Two additional mutational signatures (cyan and blue, Fig. 1) were inferred to occur most frequently in MSI-H tumors, the blue signature also appearing in tumors with deregulated activity of the polymerase Pol $\epsilon$. Deregulated polymerase activity is defined using mutational data (see [10]). The cyan signature reported a $\mathrm{C}>\mathrm{A}$ substitution occurring with a $5^{\prime} \mathrm{C}$; the blue signature identified $\mathrm{C}>\mathrm{T}$ and $\mathrm{T}>\mathrm{C}$ substitutions occurring with a $5^{\prime} \mathrm{G}$ (Fig. 1). Both of these signatures resemble signatures previously

Table 1 Cosine similarities of de-novo signatures (6 signatures in Fig. 1) with the COSMIC (May 2019) single-base substitution signatures, and with the pmSignatures from Shiraishi's paper

\begin{tabular}{|c|c|c|c|c|c|c|c|c|c|c|c|c|c|}
\hline \multirow{2}{*}{$\begin{array}{l}\text { De-novo } \\
\text { Signatures }\end{array}$} & \multicolumn{7}{|c|}{ COSMIC } & \multicolumn{6}{|c|}{ pmSignature } \\
\hline & SBS1 & SBS6 & SBS10a & SBS10b & SBS15 & SBS20 & SBS40 & 1 & 7 & 8 & 11 & 15 & 27 \\
\hline Red & 0.830 & 0.778 & 0.020 & 0.238 & 0.530 & 0.214 & 0.281 & 0.002 & 0.863 & 0.215 & 0.317 & 0.305 & 0.034 \\
\hline Orange & 0.002 & 0.014 & 0.943 & 0.260 & 0.050 & 0.087 & 0.353 & 0.991 & 0.013 & 0.015 & 0.002 & 0.139 & 0.152 \\
\hline Yellow & 0.261 & 0.207 & 0.006 & 0.914 & 0.102 & 0.025 & 0.069 & 0.001 & 0.289 & 0.971 & 0.052 & 0.084 & 0.001 \\
\hline Cyan & 0.004 & 0.042 & 0.108 & 0.041 & 0.116 & 0.884 & 0.279 & 0.175 & 0.024 & 0.002 & 0.005 & 0.173 & 0.876 \\
\hline Blue & 0.267 & 0.737 & 0.036 & 0.108 & 0.844 & 0.250 & 0.204 & 0.005 & 0.462 & 0.139 & 0.791 & 0.485 & 0.028 \\
\hline Purple & 0.157 & 0.354 & 0.270 & 0.263 & 0.322 & 0.415 & 0.911 & 0.225 & 0.379 & 0.153 & 0.159 & 0.815 & 0.292 \\
\hline
\end{tabular}


reported by Shiraishi et al. [3] in stomach cancer (pmsignatures 11 and 27 with cosine similarities of 0.79 and 0.88 , respectively, Table 1 ). The six normal ASC and MMR-proficient tumor mutation catalogs were composed primarily of the red and purple signatures. For more on these samples see Additional file 1.

We compared our new signatures to those found in the COSMIC v89 May 2019 database (Mutational Signatures v3) (Table 1). Our blue signature resembles COSMIC signature SBS15, associated with defective DNA mismatch repair (cosine similarity 0.844 ). The new cyan signature resembles SBS20, reported to be associated with combined deficiencies in DNA mismatch repair and POLD1 proofreading (cosine similarity 0.884 ).

To investigate whether the signatures we detected in the tumors varied by the time of occurrence, we refitted the mutational signature model to the subset of 93 tumors with mutations grouped separately as trunk or branch. We specified and estimated four signatures only, as none of the 93 tumors carried the Pol $\epsilon$ signatures. The results in Fig. 2 show little discernible difference in mutational signature burden between trunk and branch mutations. Indeed, the MSI tumors show no evidence of differential trunk/branch mutational burden (all signature-specific 95\% credible intervals include zero) (Additional file 1: Table S2). Interestingly, the MSS tumors show a 9.6\% higher mutational exposure of the red signature $(\mathrm{C}>\mathrm{T}$ at $\mathrm{CpG})$ in trunk compared to branch mutations (95\% credible interval: 0.047-0.114).

Finally, we sought to replicate our mutational signatures in an independent set of cancers from China. We apply the same probabilistic mutation signature model to the Chinese COCA-CN data set and identify the same six mutational signatures (Additional file 1: Figures S3, S4), replicating those extracted from the COAD-US data set. Although we lack information on tumor subtype, when ordering the tumors by the total number of mutations, a correlate for the MSI-H subtype, the pattern of estimated burdens for each mutational signature mimics those from the analysis of COAD-US cancers (see Additional file 1: Methods for details).

\section{Discussion}

We conducted a mutational signature analysis of colon adenocarcinomas from TCGA. We identified six mutational signatures using the probabilistic mutational signature model with five-base patterns, whereas an early publication only reported four [3]. The ASCs from normal colon and MMR-proficient tumors showed a mutational signature for aging, whereas the MMR-deficient tumors showed multiple MMR-related signatures.

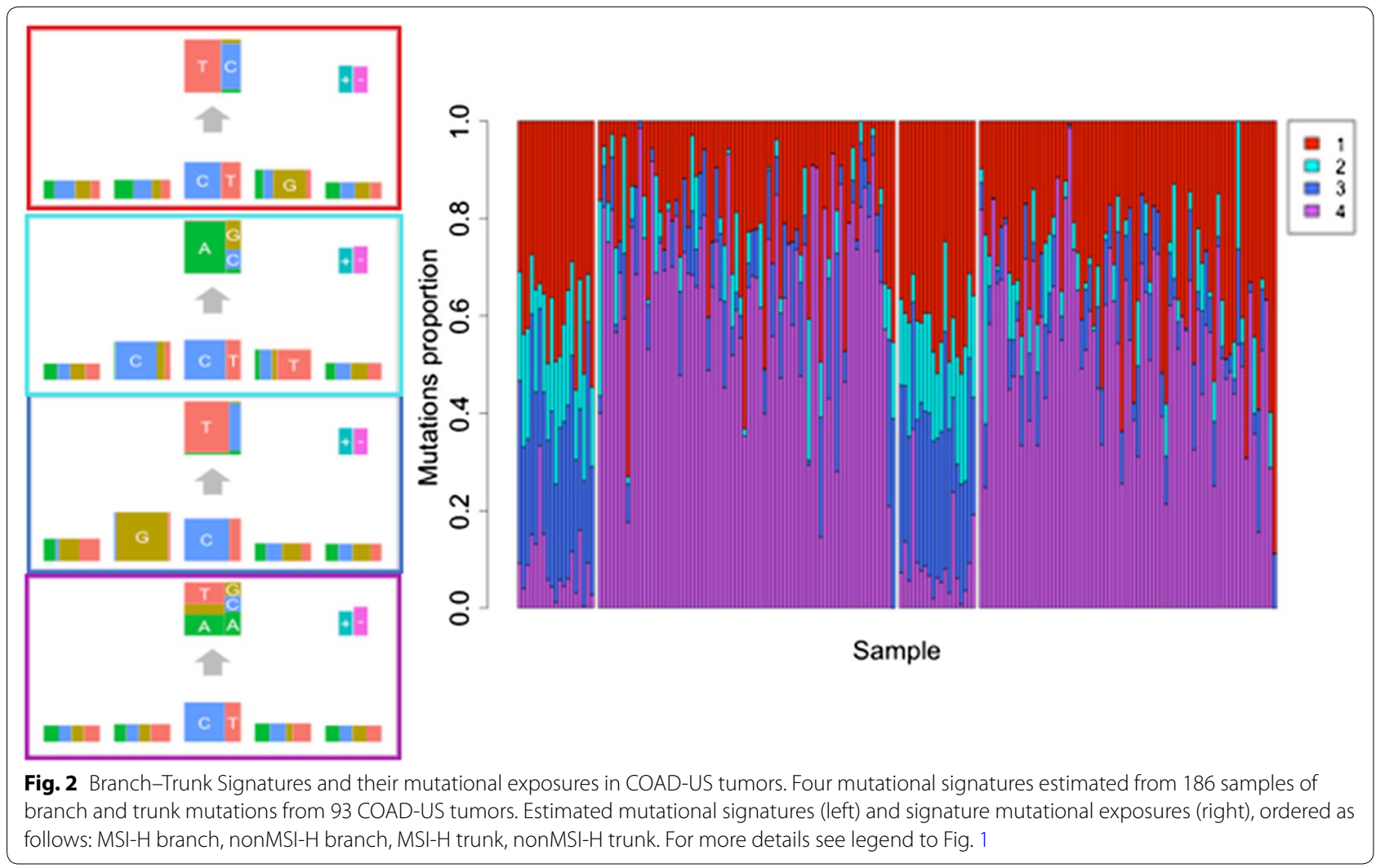


A recent paper by Liu et al. also reported six signatures but allowed only three-base patterns in a more highly parameterized model [6]. The signatures from the two approaches were slightly different. Our model pooled substitutions with similar neighboring bases into a single signature (e.g. $\mathrm{GpC}>\mathrm{GpT}$ and $\mathrm{GpT}>\mathrm{GpC}$ in Fig. 1, blue) when theirs did not. Conversely, theirs combined substitutions with different neighboring bases into a single signature $(\mathrm{CpC}>\mathrm{CpA}$ and $\mathrm{GpC}>\mathrm{GpT}$ in COSMIC signature SBS6) when ours did not. The signatures we found replicated in an independent set of Chinese COCA-CN samples.

After classifying our mutations into time of occurrence, trunk or branch, we found the signature for aging (red) was more frequent in trunk than branch mutations from MSS tumors but the same was not true for MSI tumors. This replicates the results from an earlier study of MSS colon cancers that also found a higher mutational exposure of the aging signature in trunk compared to branch mutations [14]. The lack of any new mutational signature in branch mutations, despite the different micro-environments of cancer from normal colon, is interesting.

\section{Limitations}

- TCGA published high-quality mutations from their Multi-Center Mutation Calling in Multiple Cancers (MC3) project in March 2018 [15], after the data for this paper were downloaded. The MC3 project reported variants on 389 (90\%) of our 431 cancers, identifying 104,557 (82\%) of the mutations we used for those same tumors. They identified 240585 variants, 1.9 times the number in our study. The smaller number of mutations in our analysis likely affected the precision of our estimates, and potentially also our sensitivity to detect new signatures. This limitation could be more problematic for the analysis of trunk versus branch mutations as we are likely to be differentially missing more branch than trunk mutations.

- The somatic mutation data from the Chinese COCA$\mathrm{CN}$ samples did not include variant allele frequency so we were unable to filter this data set using the same strict rules. Nevertheless, we still found evidence for the same six signatures in colon cancer, and the burdens of the new signatures in MSI-H tumors were over-represented in the tumors with high mutation burden. Therefore, despite not having information on microsatellite instability of the cancer, we can roughly infer which tumors they are based on their mutational signatures and total mutation burden. This remains to be validated.
- Our new analysis discovered a signature with a preponderance of $\mathrm{C}>\mathrm{A}$ substitutions, a common substitution for smoking, occurring at $\mathrm{CpC}$ sites. This signature appears in MSI-H tumors more frequently than MSS tumors. At the same time, epidemiologic research has found that a history of smoking is more frequent in patients with MSI-H compared to MSS tumors [16, 17]. Unfortunately, we do not have information on smoking history for COAD-US patients to investigate this.

\section{Supplementary information}

Supplementary information accompanies this paper at https://doi. org/10.1186/s13104-019-4820-0.

Additional file 1. Additional figures and tables.

\section{Abbreviations}

AIC:: Akaike Information Criterion; BIC:: Bayesian Information Criterion; COADUS::: Colon Adenocarcinoma US; COSMIC:: Catalogue of Somatic Mutations in Cancer; ICGC:: International Cancer Genomics Consortium; MMR:: mismatch repair; MSI-H:: microsatellite instable high; MSI-L:: microsatellite instable low; MSS:: microsatellite stable; TCGA:: The Cancer Genome Atlas.

\section{Acknowledgements}

The authors would like to thank the investigators from the Cancer Genome Atlas and their funding source, the National Cancer Institute, for making the COAD-US data publicly available through the Genomics Data Common (GDC) data portal. The data are from ver4.0. The authors would also like to thank Drs. Huanming Yang and Youyong Lu and their funding sources, The China Cancer Genome Consortium, The China Ministry of Science and Technology, the National High Technology Research and Development Program ("863" Program) of China and the National Natural Science Foundation of China, for making the COCA-CN publicly available through the ICGC data portal. The data are from release 27 and are made available without limitations.

\section{Authors' contributions}

The study was conceived by DS and KDS. PP downloaded all the data and performed the data analysis under the supervision of KDS and PM. The manuscript was written by PP, KDS and PM. ZY assisted with the analysis and performed critical editing. All authors read and approved the final manuscript.

\section{Funding}

This work was supported by NCI Grant Numbers P01CA196569 and P30CA014089 and NIEHS Grant Number P30ES07048. The content is solely the responsibility of the authors and does not necessarily represent the official views of the National Institutes of Health. The funding body, NIH, did not play any role in the design of the study, the collection, analysis, and interpretation of data, or in writing the manuscript.

\section{Availability of data and materials}

Only publicly available data were analyzed in this paper. The final datasets and code are available from the corresponding author upon request.

Ethics approval and consent to participate Not applicable.

\section{Consent for publication}

Not applicable.

\section{Competing interests}

The authors declare that they have no competing interests. 


\section{Author details}

${ }^{1}$ Department of Preventive Medicine, Keck School of Medicine of the University of Southern California, 2001 N. Soto Street, Los Angeles, CA 90032, USA. ${ }^{2}$ Department of Pathology, Keck School of Medicine of the University of Southern California, 2011 Zonal Ave, Los Angeles, CA 90033, USA.

Received: 30 April 2019 Accepted: 21 November 2019

Published online: 03 December 2019

\section{References}

1. Alexandrov LB, Nik-Zainal S, Wedge DC, Aparicio SA, Behjati S, Biankin AV, Bignell GR, Bolli N, Borg A, Børresen-Dale A-L, et al. Signatures of mutational processes in human cancer. Nature. 2013;500(7463):415.

2. Signatures of mutational processes in human cancer. v3-May 2019. https ://cancer.sanger.ac.uk/cosmic/signatures

3. Shiraishi Y, Tremmel G, Miyano S, Stephens M. A simple model-based approach to inferring and visualizing cancer mutation signatures. PLoS Genet. 2015;11(12):1005657.

4. Campbell BB, Light N, Fabrizio D, Zatzman M, Fuligni F, de Borja R, Davidson S, Edwards M, Elvin JA, Hodel KP, et al. Comprehensive analysis of hypermutation in human cancer. Cell. 2017;171(5):1042-56.

5. Meier B, Volkova NV, Hong Y, Schofield P, Campbell PJ, Gerstung M, Gartner A. Mutational signatures of dna mismatch repair deficiency in C. elegans and human cancers. Genome Res. 2018;28(5):666-75.

6. Liu Y, Sethi NS, Hinoue T, Schneider BG, Cherniack AD, Sanchez-Vega F, Seoane JA, Farshidfar F, Bowlby R, Islam M, et al. Comparative molecular analysis of gastrointestinal adenocarcinomas. Cancer Cell. 2018;33(4):721-35.

7. Blokzijl F, De Ligt J, Jager M, Sasselli V, Roerink S, Sasaki N, Huch M, Boymans S, Kuijk E, Prins P, et al. Tissue-specific mutation accumulation in human adult stem cells during life. Nature. 2016;538(7624):260.

8. Blokzijl F, De Ligt J, Jager M, Sasselli V, Roerink S, Sasaki N, Huch M, Boymans S, Kuijk E, Prins P, et al. Tissue-specific Mutation Accumulation in Human Adult Stem Cells During Life. https://wgs11.op.umcutrecht.nl/ mutational_patterns_ASCs/.Accessed 7 Oct 2017.
9. Genomic Data Commons Data Portal. https://portal.gdc.cancer.gov/. Accessed 03 Mar 2017.

10. Shinbrot E, Henninger EE, Weinhold N, Covington KR, Göksenin AY, Schultz N, Chao H, Doddapaneni H, Muzny DM, Gibbs RA, et al. Exonuclease mutations in DNA polymerase epsilon reveal replication strand specific mutation patterns and human origins of replication. Genome Res. 2014:24(11):1740-50.

11. Williams MJ, Werner B, Barnes CP, Graham TA, Sottoriva A. Identification of neutral tumor evolution across cancer types. Nat Genet. 2016:48(3):238.

12. International Cancer Genome Consortium (ICGC) Data Portal, ChinaColorectal Cancer. https://icgc.org/icgc/cgp/73/371/1001733. Accessed 30 May 2018

13. iMutSig: a web application to identify the most similar mutational signature using shiny. Applied to mutational signatures v3-May 2019. https:// github.com/USCbiostats/iMutSig.

14. Yang Z, Pandey P, Shibata D, Conti DV, Marjoram P, Siegmund KD. Hilda: a statistical approach to investigate differences in mutational signatures. Peer J. 2019:7:e7557. https://doi.org/10.7717/peerj.7557.

15. Ellrott K, Bailey M, Saksena G, Covington K, Kandoth C, Stewart C, Hess J, Ma S, Chiotti K, McLellan M, Sofia H, Hutter C, Getz G, Wheeler D, Ding L, MC3 Working Group, CGARN. Scalable open science approach for mutation calling of tumor exomes using multiple genomic pipelines. Cell Syst. 2018;6(3):271-81.

16. Slattery M, Curtin K, Anderson K, Ma K, Ballard L, Edwards S, Schaffer D, Potter J, Leppert M, Samowitz W. Associations between cigarette smoking, lifestyle factors, and microsatellite instability in colon tumors. J Natl Cancer Inst. 2000;92(22):1831-6.

17. Poynter JN, Haile RW, Siegmund KD, Campbell PT, Figueiredo JC, Limburg P, Young J, Le Marchand L, Potter JD, Cotterchio M, et al. Associations between smoking, alcohol consumption, and colorectal cancer, overall and by tumor microsatellite instability status. Cancer Epidemiol Prev Biomark. 2009;18(10):2745-50.

\section{Publisher's Note}

Springer Nature remains neutral with regard to jurisdictional claims in published maps and institutional affiliations.
Ready to submit your research? Choose BMC and benefit from:

- fast, convenient online submission

- thorough peer review by experienced researchers in your field

- rapid publication on acceptance

- support for research data, including large and complex data types

- gold Open Access which fosters wider collaboration and increased citations

- maximum visibility for your research: over 100M website views per year

At $B M C$, research is always in progress.

Learn more biomedcentral.com/submissions 